\title{
Choice of antidepressants by psychiatrists working with old people
}

\author{
John Wattis, Peter Bentham and John Bestley
}

In 1992, 46 consultants in old age psychiatry were asked to list their five most frequently used antidepressants, with reasons for their choices, and to comment on an information set for evaluating an antidepressant for use in older people. Lofepramine tied with dothiepin as the most popular antidepressant. The relatively new selecttve serotonin reuptake inhlbitors (SSRIs) were also widely used. Cardiovascular safety, lack of anticholinerglc side effects and sofety in overdose were important factors in choice. Some antidepressants were preferred for particular symptoms or circumstances. A modilied information set for evaluating an antidepressant for use in older people is presented.

Many factors influence the choice of an antidepressant by psychiatrists working with old people. They include efficacy, side-effect profile, toxicity in overdose, convenience of dosage regime, tablet size, availability of a liquid preparation, and cost. The introduction of new antidepressants with equivalent efficacy, lower toxicity and different side-effects profiles has led to controversy, with some advocating use of the older antidepressants largely on cost grounds and apparent lack of significant differences in side-effects (BMJ meta-analysis, Song et al, 1993) and others advocating more widespread use of the newer antidepressants such as lofepramine and the SSRIs, mostly on grounds of increased tolerability of side-effects and apparent increased safety especially in overdose (BAP consensus, British Association for Psychopharmacology, 1993).

We decided to survey a sample of consultants in old age psychiatry working in a variety of health regions to ascertain their preferences and what information they thought would be useful to them in making a logical choice between the many different antidepressants.

\section{The study}

A list of information relevant to decisions about which antidepressants to prescribe was produced by discussion between the authors and two consultant colleagues. A letter was then drafted asking consultants working with old people to list their five most commonly chosen antidepressants for use with old people and any particular circumstances (e.g. age over $\mathbf{8 0}$, cardiovascular disease) that would cause them to choose a particular antidepressant. They were also asked to comment on the information list. Consultants were not asked to put their choices in any particular order. The letter was sent by post to 28 consultant psychiatrists in old age psychiatry in the Yorkshire region and delivered by hand to 18 consultants in East Suffolk, Cambridge, Southampton, Gateshead, South Tyneside and Manchester by one of the authors (PB) who was visiting a variety of services as part of his training. The survey was carried out in spring to early summer 1992.

\section{Findings}

Fifteen of the 28 Yorkshire consultants and 17 out of 18 other consultants replied. Many put their choices in rank order but two explicitly did not and some gave particular circumstances in which they would consider a specific antidepressant. Nineteen different antidepressants were mentioned by our respondents. They are listed with frequency mentioned and frequency given as first choice in Table 1.

Lithium has been excluded from this table because it was mostly mentioned as an adjuvant therapy (by 17 respondents in all). Carbamazepine was also mentioned by two respondents as useful in difficult to treat cases, as was L-tryptophan. Although the letter did not mention ECT, three respondents specifically mentioned its importance in their practice. Comments about why people chose individual antidepressants could be classified under four categories: use for specific symptoms; avoldance of risk from side-effects or overdose; unwanted side-effects: and useful side effects. Table 2 summarises these findings.

Four commented on the use of another class of drug if one failed and several respondents gave 
Table 1. Antidepressants mentioned by correspondents in order of frequency mentioned

\begin{tabular}{ll}
\hline $\begin{array}{l}\text { Number of times } \\
\text { mentioned } \\
\text { (maximum=26) }\end{array}$ & $\begin{array}{l}\text { Antidepressant (number } \\
\text { first cholce in brackets) }\end{array}$ \\
\hline 26 & Lofepramine (9), dothiepin (8) \\
18 & Fluoxetine (1) \\
12 & Amitriptyline (6) \\
11 & Trazodone (0) \\
10 & Paroxetine (3) \\
8 & Clomipramine (0), phenelzine (0) \\
6 & Doxepin (1) \\
5 & Fluvoxamine (1) \\
4 & Sertraline (0), imipramine (0) \\
3 & Flupenthixol (0), tranylcypramine (0) \\
2 & Nortriptyline (1), mianserin (1) \\
1 & Amoxapine (0) \\
\hline & Trimipramine (0), isocarboxazid (0)
\end{tabular}

personal 'protocols' about how they chose a drug for a particular patient's characteristics.

The information preferred by old age psychiatrists in assessing a new antidepressant, revised in the light of comments from respondents, is listed in Table 3.

\section{Comment}

The response rate from the personal delivery of letters of $94 \%$ was predictably higher than from the postal survey (54\%). Lofepramine and dothiepin virtually tied as first choice. They present an interesting contrast. Dothiepin has been very widely prescribed over many years. It possesses typical tricyclic side-effects. Although there is an enduring clinical impression that side-effects are less severe than with other tricyclics this has been challenged and there is known cardiovascular toxicity in overdose (Montgomery et al, 1989). Lofepramine is also a tricyclic but with definitely reduced cardiotoxicity and toxicity in overdose and probably less pronounced side-effects than the older tricyclics (Kerihuel \& Dreyfus, 1991).

At the time of this survey the SSRIs were relatively newly launched. Despite this, fluoextine was the third most frequently mentioned although only one consultant used it as first choice. As a class the SSRIs were mentioned 37 times against 59 times for the older tricyclics. This interest is reflected in and perhaps explained by the main reason given by most respondents for choosing a particular drug or class of drug: cardiovascular risk, mentioned as a factor in $\mathbf{2 5}$ instances. This is understandable. given the close association of depressive disorder and physical illness including cardiovascular disorder (Cameron et al, 1990) and the association of physical illness with poor prognosis for depression in old age (Murphy, 1983; Baldwin \& Jolley, 1986). Suicide risk was mentioned only six times - perhaps less than one might expect. But it is likely that other methods of reducing

Table 2. Reasons given for choosing particular antidepressants (number mentioning each item in parentheses)

\section{Symptoms}

Anxiety/phobia

Atypical depression

Extra pyramidal symptoms

Psychotic symptoms

Agitation

\section{Avoldance of risk}

Cardiovascular disease

Avoid

Suicide risk present

Avoid

Sedation and falls a problem

In epilepsy

\section{Avoidance of side effects \\ Anticholinergic \\ 'Tricyclic' effects generally \\ Constipation Avoid \\ 'Useful' side effects \\ Sedation}

\author{
Phenelzine (3) \\ Monoamine oxidase inhibitors (2) \\ Paroxetine (1)*, selegiline (1)** \\ Amoxapine (1) \\ Dothiepin (1), paroxetine (1)
}

SSRIs (16), lofepramine (3), trazodone (3), nortriptyline (1)

Dothiepin (1), amitriptyline (1)

Lofepramine (3), paroxetine (1)

Dothiepin (2)

SSRIs (1), clomipramine (1), lofepramine (1)

Trazodone (1)

SSRIs (4), lofepramine (4)

SSRIs (6), lofepramine (1)

Lofepramine (2)

Dothiepin (5), amitriptyline (2), doxepin (2), trazodone (1), trimipramine (1).

"But see recent reports of acute dystonia with paroxetine (Committee on Safety of Medicines, 1993)

" depression not a recognised indication 
1. Experience and approval. How long has the drug been available on the world and UK markets? Has it been approved by the Food \& Drug Administration (USA) and if not why not?

2. Efilicacy. Double-blind placebo and reference-drug controlled trials. Long-term trials of continuation therapy and prophylactic use. Any special usefulness in old age or for conditions other than depressive disorder (e.g. obsessive-compulsive disorder).

3. Side effects. From double-blind controlled trials. List of particular side effects and expected frequency and seriousness. Yellow card reports. Recommended management.

4. Toxiclity. In overdose. In long-term use. Idiosyncratic reactions - type and incidence. Deaths per patient-year on treatment.

5. Interactions. With other drugs, including MAOls, lithium and neuroleptics specifically. With ECT.

6. Dosage regime. Is it different between old and younger people? is titration necessary? Can the product be given once daily?

7. Formulation. Range of dosages. Physical size and shape of tablets. Packaging. Slow release, liquid or parenteral formulations?

8. Pharmacokinetics and dynamics. Effective half-life and that of any active metabolites. Route of elimination.

9. Daily treatment cost at maximum recommended dose and maintenance dose in hospltal and community.

10. Information on clinical trials. Where conducted, type and phase of trial, entry and exclusion criteria, number of subjects aged over 65 years, age and gender distribution, setting, length of trial and follow-up, main measure of response to treatment and speed of response to treatment. Only trials specifically in old people or where old people were included and analysed separately.

suicide risk would be important in psychiatric practice, since prescribed antidepressants are only responsible for a minority of deaths and other methods of self-harm must be considered in planning management of the suicidal patient. Further, toxicity in overdose is often related to cardiovascular effects and lofepramine the commonest 'first-choice' drug has low cardiovascular toxicity and toxicity in overdose. This suicide risk factor might be more important in primary care where methods of assessing and reducing suicide risks may be less refined.

Specific psychiatric symptoms guiding choice of drug were mentioned ten times but the numbers for any individual symptom were small, suggesting that - with the possible exception of 'beneficial' sedation for insomniac or agitated patients, mentioned separately 11 times - this is not a very important factor in choice at present. None of our respondents mentioned the risks involved in car driving with the sedative antidepressants, whether because many patients in this age group do not drive or because the risk was not perceived as high, cannot be said.

The SSRIs and lofepramine were specifically mentioned for the avoidance of anticholinergic (eight times) and 'tricyclic' effects (seven times) although the problem of constipation with lofepramine was mentioned twice. Overall, low cardiovascular risk is the most mentioned reason for prescribing a particular drug or class of drug, beneficial sedation the second, anticholinergic effects third, and lack of tricyclic side-effects (unspecified) fourth most important.

This group of old age psychiatrists seem to have logical reasons for their choice of anti- depressants and this is reflected in the information-set they would ideally like in personally evaluating a new drug. In addition to basic data such as that on efficacy, side-effects, toxicity and interactions it is noteworthy that practical issues like formulation, frequency of dose, tablet size and packaging are also mentioned.

This study is a description of the current views of a widespread sample of old age psychiatrists on the choice of antidepressants. As such it has many limitations; but it also has the value of reflecting what really influences choice for the practical clinician working with an unselected group of patients, many of whom would probably have been excluded from clinical trials because of intercurrent physical or even mental health problems.

\section{References}

BALDWIN, R. \& JollY, D. (1986) The prognosis of depression in old age. British Journal of Psychiatry. 149, 577-583.

BRITSH ASSOCIATION FOR PSYCHOPHARMACOLOGY (Chairman: Montgomery, S.A.) (1993) Guidelines for treating depressive illness with antidepressants. Journal of Psychopharmacology. 7, 19-23.

CAMERON, O.G., RoOSE, S.P., RoBinson, R.G., KATZ, I.R. et al (1990) Perspectives on the relationship between cardiovascular disease and affective disorder-discussion. Journal of Clinical Psychiatry, 61(suppl). 10-11.

COMMITTEE ON THE SAFETY OF MEDICINES (1993) Dystonia and withdrawal symptoms with paroxetine. Current Problems (Pharmacovigilance) 19, 1.

KERIHUEL, J.C. \& DREYFUS, J.F. (1991) Meta-analysis of the efficacy and tolerability of the tricyclic antidepressant Lofepramine. Journal of International Medical Research, 19, 183-201.

MONTGOMERY, S.A., BALDWIN, D. \& GREEN, M. (1989) Why do amitriptyline and dothiepin appear to be so dangerous 
in overdose? Acta Psychiatrica Scandinavica, 80/suppl 1354), 47-54.

MURPHY, E. (1983) The prognosis of depression in old age. British Journal of Psychiatry, 142, 111-119.

Song. F., Freemantle. N., Sheldon. T.A., House. A. et al (1993) Selective serotonin reuptake inhibitors: metaanalysis of efficacy and acceptability. British Medical Journal, s06, 683-687.
John Wattis, Consultant and Sentor Lecturer in Psychiatry for Old People, Bridge House, Balm Road, Leeds LS10 2TP; Peter Bentham, Consultant in Psychiatry for Old People, Queen Elizabeth Hospital, Birmingham, B15 2Q2; and John Bestley. Senior Registrar in Psychiatry for Old People, Yorkshire Training Scheme

\title{
Psychiatric admission services for people with learning disability
}

\author{
I. Singh, M.I. Khalid and M.J. Dickinson
}

\begin{abstract}
As the care of people with learing discbillty has shifted from large Victorion hospliats to the community, provision for in-patient poychiatic treatment has, in many districts, also moved. Pupose bullt district or supradistrict admission services represent the most common model. An alternative is the use of existing general poychiatilic beds. In this article we describe the first 18 months of the Hillingdon district service where this latter model has been adopted.
\end{abstract}

The last 20 years have seen a significant change in models of psychiatric service for people with learning disability. Until recently the majority of individuals requiring admission went to large hospitals typically situated on the periphery of major towns where there was also provision for residential care. Many of these are now closing, or have closed, with districts developing alternative community facilities.

In North West Thames purpose-built community units serving single districts have appeared and there is at least one supra district service, based in the community, offering psychiatric admission facilities in learning disability from its three surrounding districts, as well as encouraging extra contractual business. An alternative strategy is to use existing adult psychiatry beds.

We would like to describe the first 18 months of the Hillingdon district service where, following agreement with medical, nursing and managerial groups, admission facilities for people with psychiatric illness and learning disability were made available on a general psychiatric ward at the local district general hospital (DGH). The service was to be supported by weekly consultant ward rounds, senior registrar visits and, if necessary, Leavesden Hospital, the large Victorian hospital that historically served Hillingdon along with other north London areas.

\section{The study}

Notes on all admissions to Hillingdon DGH for the psychiatric care of people with learning disability were collected between June 1991 and December 1992, (the first 18 months of this service). Records were taken of age, sex, length of stay, reason for admission, past psychiatric history, level of handicap, diagnosis (DSM-III-R) and treatment, in addition to social factors identified as important in the admission/discharge processes of this group (Dickinson \& Singh. 1991).

\section{Findings}

There were 13 admission episodes during this period, comprising 11 individuals, five men and six women, average age 38 . None of the men but two of the women were married.

The main reason for admission was following overdose/suicidal ideation, ( 3 out of 5 men, 3 out of 6 women), or aggression, ( 2 out of 5 men, 3 out of 6 women). Average length of stay was eight weeks. Two men and one woman were admitted under a section of the Mental Health Act, a third man requiring detention during his admission. All 11 individuals had a past psychiatric history. 